\title{
Mycosis Fungoides as a Cause of Severe Obstructive Sleep Apnea
}

\author{
Marco Luigetti ${ }^{1}$, Alessandro Cianfoni ${ }^{2}$, Emanuele Scarano ${ }^{3}$, Luca Laurenti ${ }^{4}$, \\ Idanna Innocenti ${ }^{4}$, Elisa Testani ${ }^{1}$, Serena Dittoni ${ }^{1}$ and Giacomo Della Marca ${ }^{1}$
}

\begin{abstract}
We report the case of a 38-year-old woman, affected by a cutaneous form of mycosis fungoides (MF) who presented with a history of loud snoring associated with sleep apnea. A polysomnographic study confirmed the presence of severe obstructive sleep apnea syndrome (OSAS). Cranial and neck MRI revealed a neoplastic infiltration of the tongue base and the posterior pharynx wall. Upper airway neoplastic infiltration is rarely reported as a cause of OSAS and extra-cutaneous localizations of MF are uncommon. This is the first case in the literature of a patient with nocturnal polysomnogram documented OSAS caused by a mucosal involvement of MF.
\end{abstract}

Key words: OSAS, mycosis fungoides, MRI

(Intern Med 50: 1753-1755, 2011)

(DOI: 10.2169/internalmedicine.50.5541)

\section{Introduction}

Obstructive sleep apnea syndrome (OSAS) is defined as a disorder of breathing during sleep characterized by prolonged partial airway obstruction and/or intermittent complete obstruction (obstructive apnea) that interrupts normal ventilation during sleep and normal sleep patterns (1).

The main risk factors for OSAS in adults are obesity and male sex, which are related to the propensity for repetitive upper airway collapse (2). Mycosis fungoides (MF), an extranodal non-Hodgkin's lymphoma, is the most common form of cutaneous T-cell lymphoma, typically of the CD4 helper T-cell phenotype $(3,4)$. We describe a patient, affected by a cutaneous form of MF, with a severe OSAS in which cranial and neck MRI revealed a neoplastic infiltration of the tongue base and the posterior pharynx wall.

\section{Case Report}

A 38-year-old woman came to our department for snoring and daytime sleepiness. The cutaneous form of MF had been diagnosed at age 27. Snoring and respiratory pauses were reported by her bed partner since age 35 . At the time of our observation, body mass index was $24.6 \mathrm{~kg} / \mathrm{m}^{2}$, neck circumference was $37 \mathrm{~cm}$, Epworth Sleepiness Scale score was 18. No diurnal respiratory impairment was present. Full night, laboratory-based video-polysomnography showed intermittent snoring and continuous, long lasting (mean duration=34.5 \pm 13.3 seconds) obstructive apneas and hypopneas, time in bed $=522$ minutes, sleep efficiency $=76.1 \%$, apnea-hypopnea index $=67.5$ events/hour, desaturation index $=$ 71.4 events/hour, lowest $\mathrm{SpO}_{2}=64 \%$. These findings confirmed the diagnosis of severe OSAS (1). Dermatological evaluation disclosed an erythematous plaque on the left lip; otolaryngological evaluation revealed thickness of the left oral mucosa. Face and neck MR imaging demonstrated a neoplastic infiltration of the tongue base and posterior pharynx wall (Fig. 1A-E); neck ultrasound confirmed the presence of an enlargement of lymph nodes; total body contrastenhanced CT scan was unremarkable. A lip biopsy was performed confirming a secondary localization of MF; bone marrow needle aspiration and biopsy revealed a medullary infiltration. Considering the severity of OSAS a treatment with continuous positive airway pressure (CPAP) was started resulting in a great improvement of respiratory disturbance.

${ }^{1}$ Department of Neuroscience, Catholic University, Italy, ${ }^{2}$ Neuroradiology Section, Department of Radiology-MUSC-Medical University of South Carolina, USA, ${ }^{3}$ Institute of Otorhinolaryngology, Catholic University, Italy and ${ }^{4}$ Institute of Haematology, Catholic University, Italy Received for publication March 22, 2011; Accepted for publication April 27, 2011 Correspondence to Dr. Marco Luigetti, mluigetti@gmail.com 

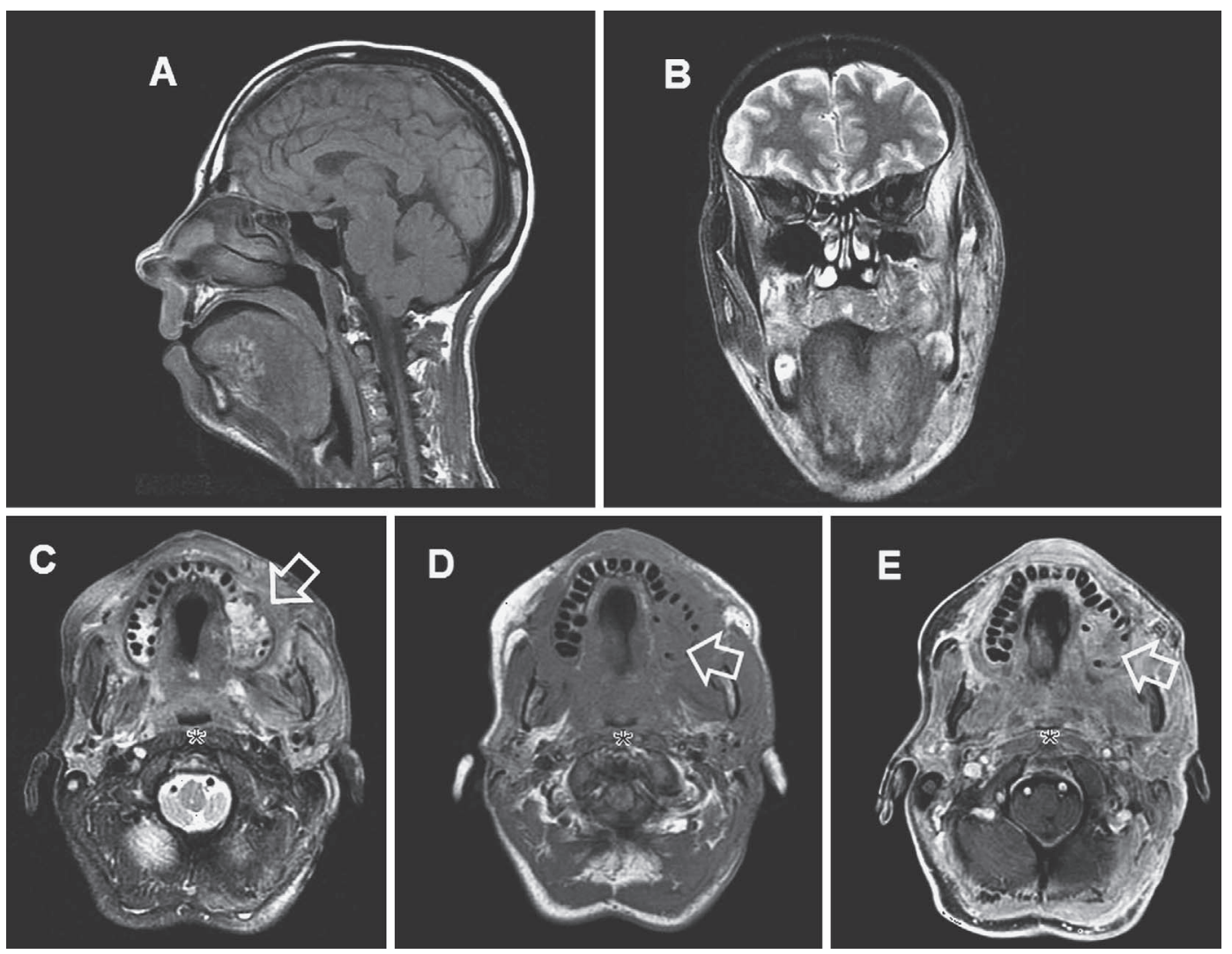

Figure 1. parts A-E: Contrast-enhanced MRI of the face. Selected images from non-enhanced sagittal T1-W (A), fat-suppressed coronal T2-W (B), fat-suppressed axial T2-W (C), non-enhanced axial T1-W (D), and fat-suppressed-enhanced axial T1-W (E), showing the striking extent of the infiltrative process, with hypointense $\mathrm{T} 1$ signal, hyperintense $\mathrm{T} 2$ signal, and diffuse contrast-enhancement. The abnormal tissue infiltrates the bone of the left maxilla, that appears expanded (better seen on C-E, white arrows), the tongue, markedly enlarged (evident on A and B), and all the soft tissues of the face and neck, superficial and deep fat planes, masticator muscles, soft palate, left parotid gland, all scarcely recognizable (see B-E). The posterior airway space appears markedly reduced (asterisk in $\mathrm{C}$-E); on the sagittal plane the distance between the posterior surface of the tongue base and the posterior wall of the pharynx is nearly abolished. No intra-cranial involvement was seen. To note on E, the diffuse abnormal contrast-enhancement of the bone and soft tissues of the face and neck, stand-out against the low-signal background created by the fat suppression technique.

Follow-up laboratory based video-polysomnography, after one month, disclosed a significant amelioration of the sleeprelated respiratory pattern (time in bed $=473$ minutes, sleep efficiency $=91.8 \%$, apnea-hypopnea index $=13.8$ events/hour, desaturation index=14.8 events/hour, lowest $\mathrm{SpO}_{2}=76 \%$ ). For the relapse of hematological disease the patient was treated with Mi.C.M.A. polychemotherapy (mitoxantron; carboplatin; aracytine; methylprednisolone). After six months, because of progressive disease, the patient underwent reduced intensity allogeneic hematopoietic stem cell transplantation as consolidation therapy. Furthermore a cycle of radiotherapy on the skin lesions was performed. New bone marrow needle aspiration and biopsy after therapy were unremarkable. Follow-up total body contrast-enhanced CT scan, after one year, detected no abnormalities; face and neck MRI showed no new lesions and confirmed those previously revealed which showed a reduction in size. The patient and her bed partner reported a decrease of snoring and respira- tory pauses. A follow-up polysomnographic study, performed by means of ambulatory cardio-respiratory monitoring, without positive pressure ventilation, disclosed a marked improvement of sleep-related respiratory pattern (time in bed=587 minutes, apnea-hypopnea index $=2$ events/ hour, desaturation index $=6$ events/hour, lowest $\mathrm{SpO}_{2}=77 \%$ ).

The patient was periodically evaluated by a hematologist every six months. At the last follow-up, after three years, the patient was in continuous clinical remission.

\section{Discussion}

We described a patient with severe OSAS since the age of 35. Clinical history revealed a cutaneous form of MF diagnosed at the age of 27 . Considering anamnestic data a cranial and neck MRI was performed. Images showed a neoplastic infiltration of the tongue base and posterior pharynx wall causing a marked reduction of posterior airways space 
and predisposing to upper airway collapse during sleep (Fig. 1A-E).

MF generally involves the skin with lesions that have a predilection for non-sun-exposed areas although any area of the skin may be affected. Extra-cutaneous localizations are uncommon, and oral mucosal involvement is rare; only a few cases have been described in which the oral cavity and the hard palate are involved by the disease (5).

The association of OSAS with tumours of the neck is not frequent $(6,7)$. In all reported cases the neck tumour caused a reduction of posterior airway space, defined as the distance between tongue base and posterior pharynx wall, that is the most important predictive parameter of OSAS (1). An improvement of respiratory symptoms after the therapy (surgery or chemotherapy) of the neck tumour is documented in all cases reported, including the present case $(6,7)$.

In conclusion upper airway neoplastic infiltration is a possible cause of severe sleep disordered breathing. This report is the first case in the literature of a patient with nocturnal polysomnogram documented OSAS caused by a mucosal involvement of MF; it underlines the importance of clinical history for a prompt diagnosis.
The authors state that they have no Conflict of Interest (COI).

\section{References}

1. Sleep-related breathing disorders in adults: recommendations for syndrome definition and measurement techniques in clinical research. The Report of an American Academy of Sleep Medicine Task Force. Sleep 22: 667-689, 1999.

2. Redline S, Kump K, Tishler PV, Browner I, Ferrette V. Gender differences in sleep disordered breathing in a community-based sample. Am J Respir Crit Care Med 149: 722-726, 1994.

3. Weinstock MA, Gardstein B. Twenty-year trends in the reported incidence of mycosis fungoides and associated mortality. Am J Public Health 89: 1240-1244, 1999.

4. Girardi M, Heald PW, Wilson LD. The pathogenesis of mycosis fungoides. N Engl J Med 350: 1978-1988, 2004.

5. Chua MS, Veness MJ. Mycosis fungoides involving the oral cavity. Australas Radiol 46: 336-339, 2002.

6. Gómez-Merino E, Arriero JM, Chiner E, Signes-Costa J, Marco J. Obstructive sleep apnea syndrome as first manifestation of pharyngeal non-Hodgkin's lymphoma. Respiration 70: 107-109, 2003.

7. Alobid I, Benítez P, Berenguer J, Bernal-Sprekelsen M, Mullol J. Parapharyngeal angiolipoma causing obstructive sleep apnoea syndrome. Acta Otolaryngol 124: 210-212, 2004.

(C) 2011 The Japanese Society of Internal Medicine

http://www.naika.or.jp/imindex.html 